\title{
Challenges for cellular endocrinology: improving the detection methods and closing the gap between in vitro and in vivo observations
}

\section{Ralf Jockers* \\ Department of Endocrinology, Metabolism and Cancer, Institut Cochin, Paris, France \\ ${ }^{*}$ Correspondence: ralf.jockers@inserm.fr}

The "Cell Theory" constitutes a fundamental element of modern biology stating that the Cell is the basic structural and functional unit of life. Cellular Endocrinology focuses on two types of cells, those specialized in the synthesis, storage and secretion of hormones and those responding to these hormones by expressing the corresponding molecular targets, called receptors. The 20th century has seen unprecedented progress in our understanding of cellular processes in particular due to the development of immortalized cell lines that have a stable phenotype and can be cultured under constant and reproducible conditions. These cell lines express either all components endogenously, or may be complemented with the protein(s) of interest by transfecting the corresponding $\mathrm{CDNA}(\mathrm{s})$. Although this approach was very successful in the past and is likely to continue to provide important findings in the future it also suffers from several limitations: (i) important features of cells might be lost upon immortalization and clonal selection of cell lines; (ii) (over)expression of exogenous proteins might activate signaling pathways that are not relevant in an in vivo context; (iii) expression of proteins in different cellular contexts produces sometimes confusing or contradicting results; (iv) different receptors activating apparently identical signaling pathways might have completely different final outcomes and we do not really understand why.

Below I will give a non-exhaustive list of ideas that might help to overcome at least some of these limitations and challenges.
We have learned from the past that the improvement of detection methods and tools is often a prerequisite for novel discoveries. Increasing the resolution of our methods to dissect more precisely the kinetics and subcellular localization of intracellular events should provide us with new information on the subcellular level and might reveal previously unappreciated functional differences. FRET biosensors to monitor intracellular second messengers in living cells in real time by FRET microscopy represent only one of these promising new tools.

Mimicking more accurately the physiological and pathological stimulation pattern of receptors will be a further challenge. Whereas current stimulation protocols are often based on the treatment of starved cells with saturating doses of a single hormone, in a natural environment, cells are rather exposed to a cocktail of hormones present at various concentrations. Cross-talks between different hormonal systems are increasingly reported in the literature and have to be taken into account in future research to fully understand the effect of hormonal stimulation in an in vivo context. In addition, differences in the temporal pattern of hormone stimulation should be taken into account. Whereas many current protocols are based on acute stimulation, prolonged, or rhythmic stimulation patterns are often seen in a natural environment. Indeed, many biological processes are under circadian control including hormonal production/metabolism as well as the synthesis of receptors and components of their signaling pathways.

To narrow the gap between in vitro and in vivo observation on the cellular level, new methods such as two-photon excitation microscopy have been developed to monitor single cells in an complex biological environment, i.e., the tissue of a living animal. The application of these techniques to animal models for endocrine diseases (cancer, obesity, type 2 diabetes, etc.) opens exciting perspectives.

Finally, future research has to scope with recent advances in molecular genomics and proteomics. These techniques have generated, and still generate, huge amounts of information relevant for Cellular Endocrinology. It is not possible anymore to handle these data with classical methods and new more global and systematic approaches have to be applied. This is where network analysis and systems biology accompanied by mathematical modeling of cellular networks are entering the stage.

Received: 12 September 2010; accepted: 21 September 2010; published online: 25 October 2010.

Citation: Jockers R(2010) Challenges for cellular endocrinology: improving the detection methods and closing the gap between in vitro and in vivo observations. Front. Endocrin. 1:3. doi: 10.3389/fendo.2010.00003

This article was submitted to Frontiers in Cellular Endocrinology, a specialty of Frontiers in Endocrinology. Copyright (c) 2010 Jockers. This is an open-access article subject to an exclusive license agreement between the authors and the Frontiers Research Foundation, which permits unrestricted use, distribution, and reproduction in any medium, provided the original authors and source are credited. 